\title{
Acute Myocardial Infarction Due to Coronary Occlusion Caused by a Metastatic Cardiac Tumor Arising from Squamous Cell Lung Cancer: An Evaluation with Three-dimensional Transthoracic Echocardiography
}

Miho Kuramoto ${ }^{1}$, Masako Okada ${ }^{2}$, Hajime Saeki ${ }^{1}$, Yasuyuki Yoshida ${ }^{3}$ and Shinji Hasegawa ${ }^{1}$

\begin{abstract}
:
A 60-year-old man developed acute myocardial infarction from the total occlusion of the right coronary artery via metastatic squamous lung cancer and was treated with percutaneous coronary intervention (PCI). Computed tomography and transthoracic echocardiography (TTE) revealed a metastatic tumor, and threedimensional TTE was useful for determining the size and location of the tumor in relation to the coronary artery. Six months after PCI, the patient died, and an autopsy confirmed extensive metastasis to the heart and nearby vessels as detected by three-dimensional TTE. Although rare, lung cancer metastasis to the heart may directly occlude the coronary artery.
\end{abstract}

Key words: acute myocardial infarction, coronary occlusion, lung cancer, cardiac metastasis

(Intern Med Advance Publication)

(DOI: 10.2169/internalmedicine.7580-21)

\section{Introduction}

Most cardiac tumors are secondary to lung, breast, and esophageal cancers, malignant lymphoma, leukemia, or malignant melanoma (1). However, since many cases are asymptomatic, incident cardiac metastasis is considered to be underestimated, and cardiac metastasis might be observed in up to $25 \%$ of patients who die of malignancies at autopsy (1).

Metastasis to the heart may produce diverse symptoms and signs, and of these, ST segment elevation on electrocardiography (ECG) requires an emergency diagnosis to determine the presence of acute myocardial infarction (AMI), which may require percutaneous coronary intervention (PCI) (2-4).

We herein report a case of a large cardiac metastatic mass from a primary lung cancer. AMI was caused by coronary artery occlusion and was successfully treated with emergency PCI. Spatial information of the metastatic tumor in the heart was derived with two-dimensional (2D) and threedimensional (3D) transthoracic echocardiography (TTE) and computed tomography (CT).

\section{Case Report}

\section{Presenting illness}

A 60-year-old Japanese man visited our hospital because of dyspnea on exertion, and he was admitted. Two years earlier, he had been diagnosed with squamous cell carcinoma of the left hilum involving the left mediastinum and been treated with radiation and chemotherapy with partial remission. However, five months before the current admission, the patient was newly diagnosed with gastric cancer (moderately to poorly differentiated tubular adenocarcinoma). Chest plain CT revealed slight pericardial effusion, and recurrence of lung cancer with metastasis to the pericardium or heart was considered to coexist with gastric cancer. Positron emission tomography and detailed TTE were not performed.

${ }^{1}$ Department of Cardiology, Japan Community Healthcare Organization Osaka Hospital, Japan, ${ }^{2}$ Department of Clinical Laboratory, Japan Community Healthcare Organization Osaka Hospital, Japan and ${ }^{3}$ Department of Pathophysiology, Japan Community Healthcare Organization Osaka Hospital, Japan

Received: March 19, 2021; Accepted: May 23, 2021; Advance Publication by J-STAGE: July 10, 2021

Correspondence to Dr. Miho Kuramoto, kuramoto-miho@osaka.jcho.go.jp 
A

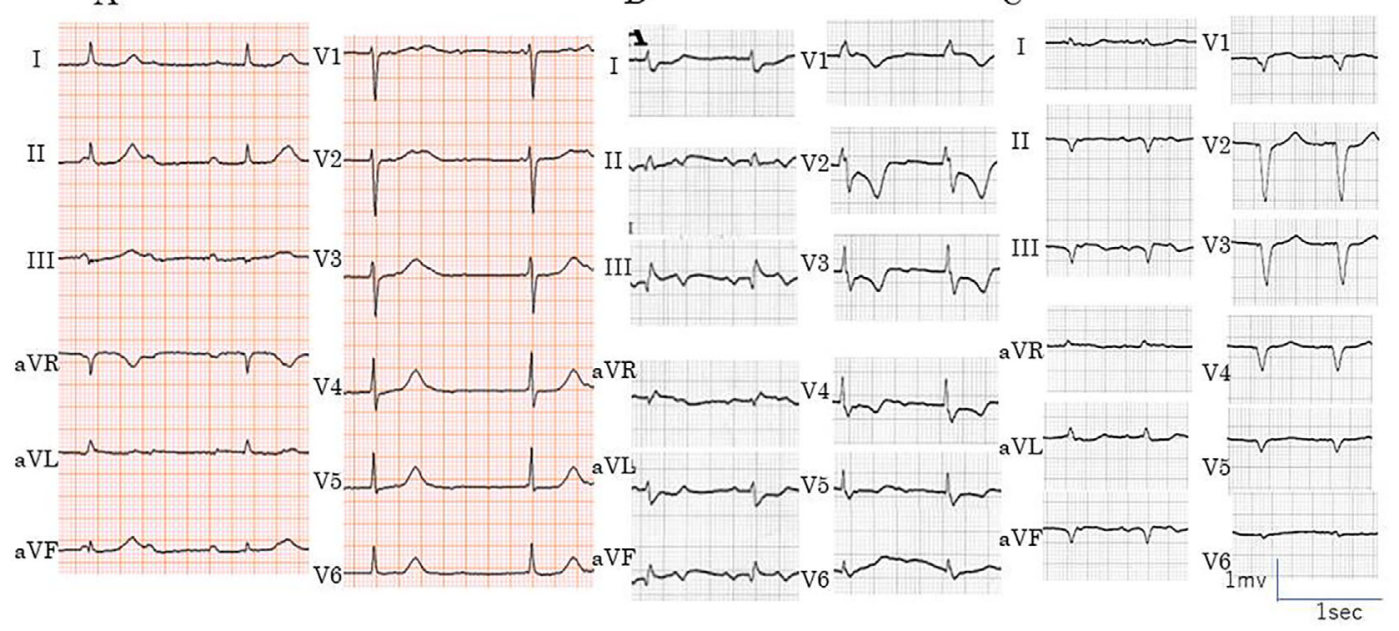

Figure 1. Findings of 12-lead electrocardiography (ECG) before admission and on admission. (A) ECG shows complete atrio-ventricular block with narrow QRS complexes. No ST-T changes were detected at this time. (B) ECG revealed atrial tachycardia (arrows) with atrio-ventricular block. STsegment elevation in leads II, III, and aVf and ST-segment depression in leads I, aVL, and V2-V6 were noted. The pacing rate was reduced to $60 \mathrm{ppm}$. (C) At the end of PCI, ST-segment remained slightly elevated in leads II, III, and aVF. Due to his unstable hemodynamics, the patient was paced at 76 ppm.

Table. Laboratory Data on Admission.

\begin{tabular}{|c|c|c|}
\hline Variable & $\begin{array}{c}\text { Reference Range, } \\
\text { Adults }\end{array}$ & On Admission \\
\hline Hematocrit (\%) & $42.0-54.0$ & 34.8 \\
\hline Hemoglobin (g/dL) & $14.0-17.0$ & $11.2 \downarrow$ \\
\hline White-cell count $(/ \mu \mathrm{L})$ & $4,000-8,500$ & 8,800 \\
\hline \multicolumn{3}{|l|}{ Differential count $(\%)$} \\
\hline Neutrophils & $40-71$ & $94.0 \uparrow$ \\
\hline Lymphocytes & $27-46$ & 3.0 \\
\hline Monocytes & $3-7$ & 3.0 \\
\hline Platelet count $\left(\times 10^{4} / \mu \mathrm{L}\right)$ & $15-35$ & $42.6 \uparrow$ \\
\hline Red-cell count $\left(\times 10^{4} / \mu \mathrm{L}\right)$ & $460-540$ & 469 \\
\hline Mean corpuscular volume (fL) & $85-100$ & $74.2 \downarrow$ \\
\hline Sodium $(\mathrm{mEq} / \mathrm{L})$ & $135-145$ & 137 \\
\hline Potassium $(\mathrm{mEq} / \mathrm{L})$ & $3.5-5.0$ & 4.9 \\
\hline Chloride $(\mathrm{mEq} / \mathrm{L})$ & $95-110$ & 103 \\
\hline Albumin (g/dL) & $3.8-5.3$ & $3.5 \downarrow$ \\
\hline Total protein $(\mathrm{g} / \mathrm{dL})$ & $6.7-8.3$ & $6.4 \downarrow$ \\
\hline Troponin I & Negative & Positive \\
\hline B-type natriuretic peptide $(\mathrm{pg} / \mathrm{mL})$ & $<18.4$ & $339.3 \uparrow$ \\
\hline C-reactive protein $(\mathrm{mg} / \mathrm{dL})$ & $<0.3$ & $3.34 \uparrow$ \\
\hline Total bilirubin (mg/dL) & $0.2-1.2$ & 0.5 \\
\hline Aspartate aminotransferase (IU/L) & $13-33$ & $68 \uparrow$ \\
\hline Alanine aminotransferase (IU/L) & $6-30$ & 13 \\
\hline Lactate dehydrogenase (IU/L) & $119-220$ & 453 \\
\hline Cholinesterase (IU/L) & $200-440$ & 223 \\
\hline Blood urea nitrogen $(\mathrm{mg} / \mathrm{dL})$ & $8-20$ & 18 \\
\hline Creatinine (mg/dL) & $0.4-1.1$ & 0.66 \\
\hline Creatinine kinase (IU/L) & $62-287$ & $618 \uparrow$ \\
\hline Creatinine kinase-MB (IU/L) & $0-25$ & $133 \uparrow$ \\
\hline
\end{tabular}

The values in blue and red are below and above the normal range, respectively. 


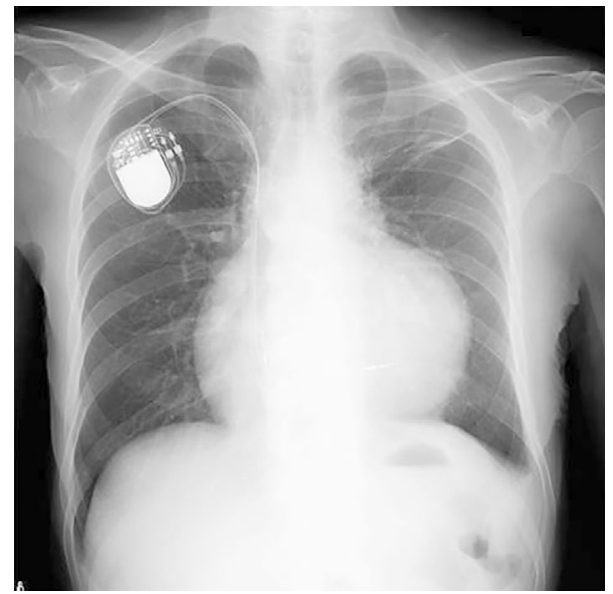

Figure 2. Findings of chest radiography on admission. Chest radiography revealed cardiomegaly, pleural indentation on the left upper lobe, and a dull costophrenic angle in the left lung. The cardiothoracic ratio was $53 \%$, and the cardiac silhouette suggested the presence of pericardial effusion.
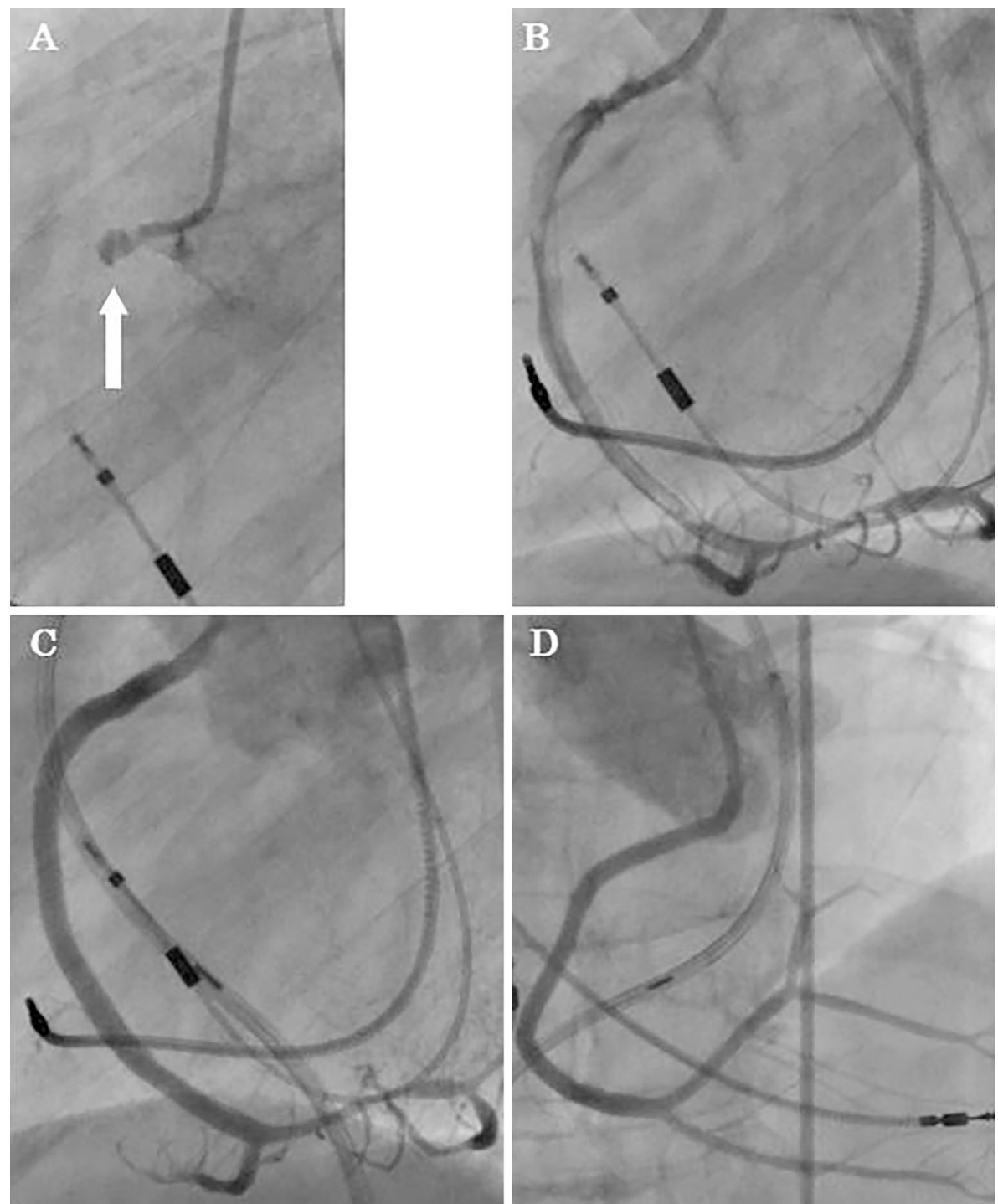

Figure 3. Findings of coronary angiography (CAG). (A) CAG on admission showed total occlusion of the proximal right coronary artery (RCA; white arrow). (B) CAG after balloon dilatation showed reprefusion but with RCA dissection. (C, D) Normal CAG findings after stent placement. 


\section{Chamber view}
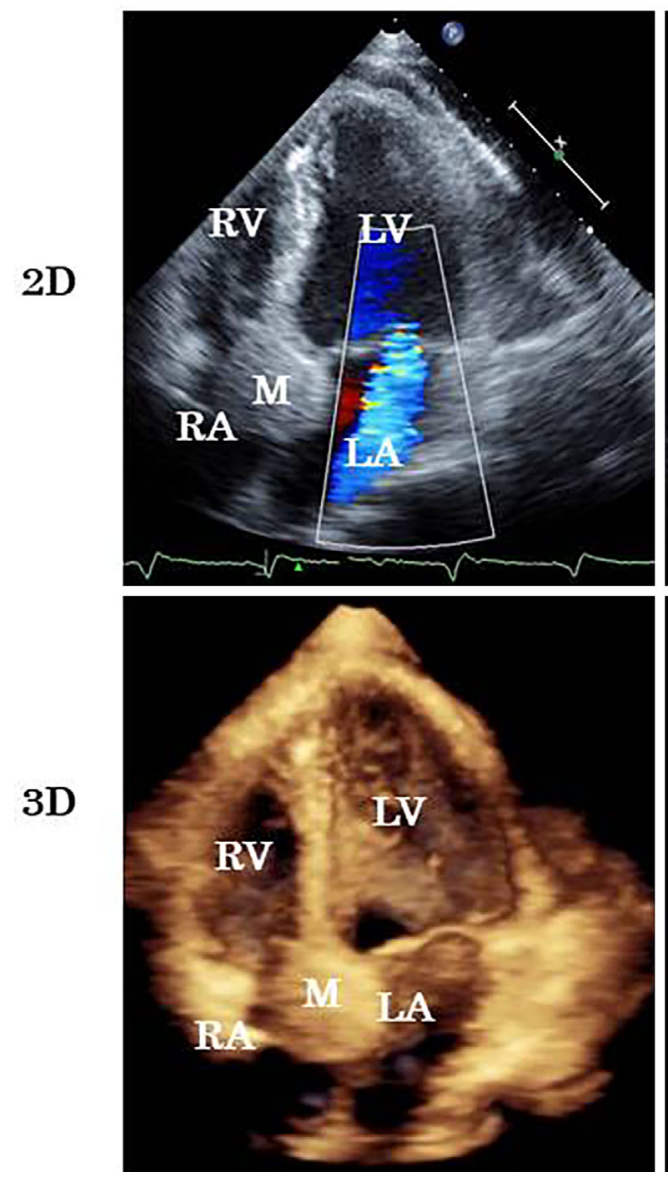

\section{Para Sternal Short Axis}
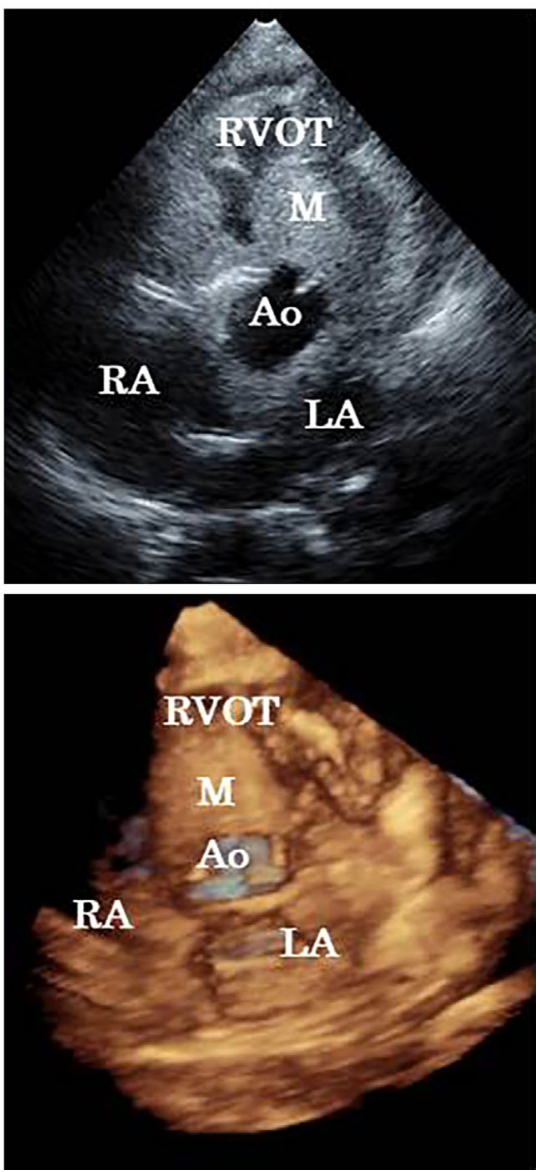

Figure 4. Two-dimensional (2D) and three-dimensional (3D) transthoracic echocardiography. Fourteen days after acute myocardial infarction, 2D images $(A, B)$ and 3D images $(C, D)$ revealed a huge mass in the right atrium over the atrioventricular septum. The stent in the right coronary artery is evident (arrows). Ao: aorta, LA: left atrium, LV: left ventricle, M: mass, RA: right atrium, RV: right ventricle, RVOT: right ventricular outflow tract

A cardiac examination revealed a holosystolic murmur (Levine II/VI) at the apex. Blood tests showed elevated cardiac enzyme and B-type natriuretic peptide levels (Table).

ECG revealed atrial tachycardia and ventricular pacing. When the pacing rate was reduced to $60 \mathrm{ppm}$, ECG showed 2-1 atrioventricular block with atrial tachycardia of 136 bpm. ST-segment elevation was noted in leads II, III, and $\mathrm{aVF}$, and reciprocal ST depression was noted in leads I, aVL, and V2-6 (Fig. 1B).

Chest radiography showed mild cardiomegaly with a cardiothoracic ratio of $53 \%$. The cardiac silhouette suggested the presence of pericardial effusion. Pleural indentation at the left upper lobe and a dull costophrenic angle were observed in the left lung (Fig. 2). 2D TTE showed a wall motion abnormality from the basal left ventricular septum to the posterior wall. Moderate mitral regurgitation and moderate pericardial effusion were also detected without signs of cardiac tamponade.

During cardiac catheterization, coronary angiography (CAG) revealed total occlusion of the right coronary artery (Fig. 3), but the left coronary artery was normal. Subse- quently, PCI was performed. The cause of the MI was consistent with occlusion by thrombus from IVUS images and coronary angiography. Repeated ballooning of the right coronary artery lesion was followed by re-occlusion and finally accompanied by dissection, and three stents were placed to restore full patency. Following successful PCI, the ST-segment elevation in leads II, III and aVF and depression in I and aVL were improved and then declined to the baseline (Fig. 1C). The patient was paced at $76 \mathrm{ppm}$ because of unstable hemodynamics. The patient was classified as Forrester subset IV, with a pulmonary capillary wedge pressure of $25 \mathrm{mmHg}$ and a cardiac index of $1.6 \mathrm{~L} / \mathrm{min} / \mathrm{m}^{2}$, and intraaortic balloon pumping was employed.

At the end of PCI, CK/CKMB peaked at 1,466/237 IU/1 and then declined towards normal levels. After PCI, the STsegment stayed elevated in leads II, III, and aVF and then declined. Due to his unstable hemodynamics, the patient was paced at $76 \mathrm{ppm}$. Following care in the coronary care unit for eight days, the hemodynamics stabilized, and the patient was moved to the cardiology ward.

On the 14th hospital day, we performed 3D TTE, which 

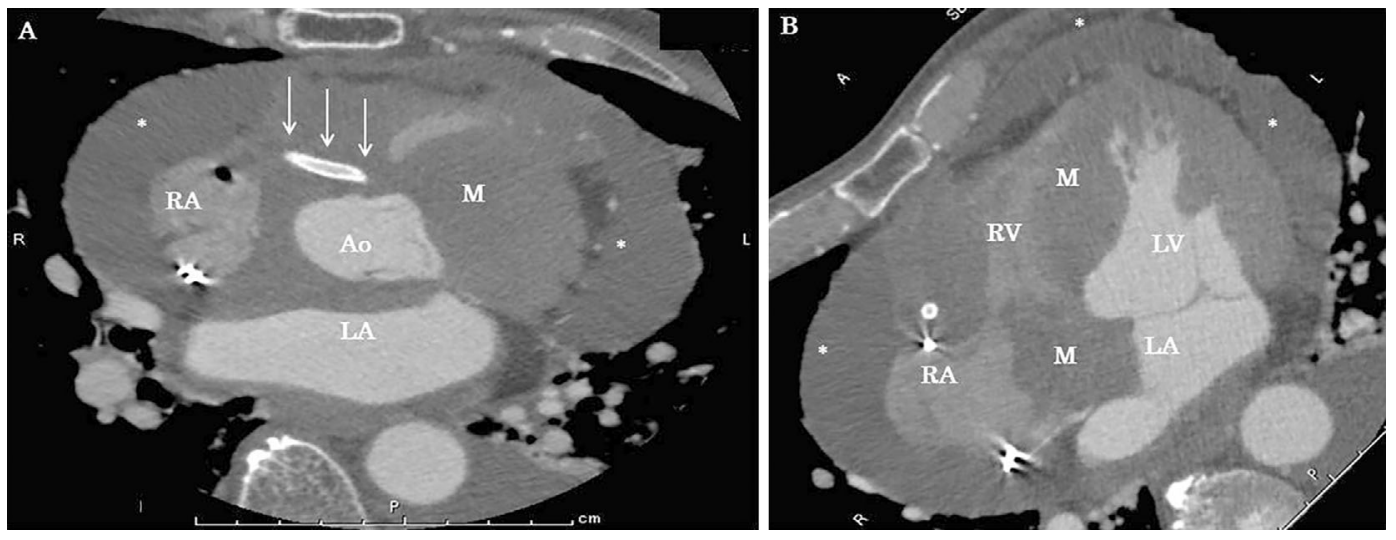

Figure 5. Contrast-enhanced computed tomography (CT). CT on day 14 after acute myocardial ischemia showed a right coronary stent running through the abnormal mass. The white arrows indicate the stent, and the asterisks represent pericardial effusion. Ao: aorta, LA: left atrium, LV: left ventricle, $M$ : mass, $R A$ : right atrium, $R V$ : right ventricle
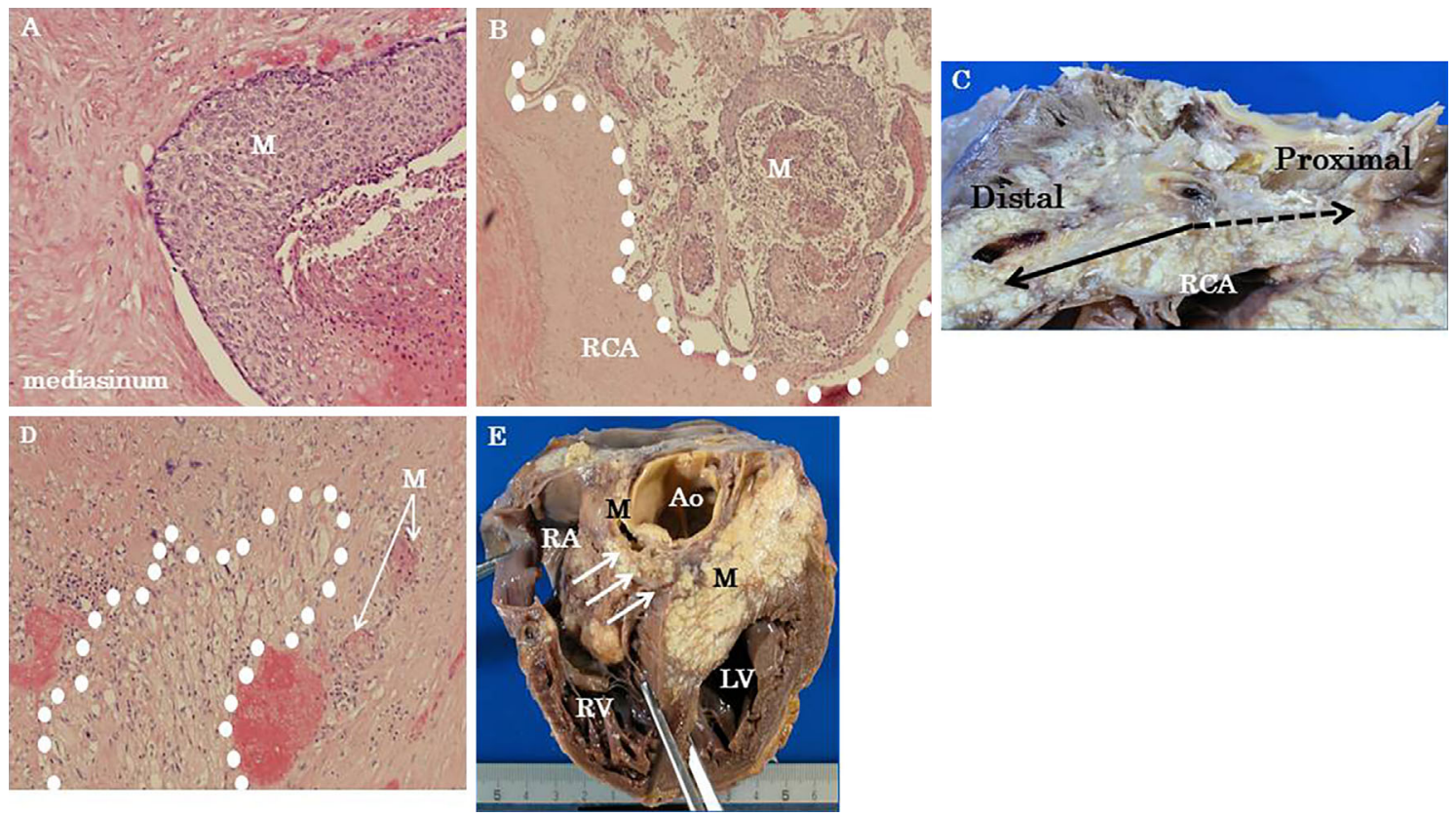

Figure 6. Autopsy findings for the heart. (A) Metastasis from squamous cell lung carcinoma invading the heart, pericardium, and mediastinum [Hematoxylin and Eosin $(\mathrm{H} \& \mathrm{E})$ staining, $\times 100$ magnification]. (B) The metastatic tumor invaded the right coronary artery (RCA) (H\&E staining, $\times 40$ magnification). (C) Gross photograph of the metastatic carcinoma showing RCA involvement. The proximal site of the RCA was invaded by the metastatic tumor and was difficult to identify. The distal RCA site was intact. (D) Metastatic lung carcinoma with conduction system involvement. Purkinje fibers were surrounded with white dots (H\&E staining, $\times 100$ magnification). (E) Gross photograph of a metastatic tumor invading the heart. The arrows indicate the location of the atrioventricular node. Ao: aorta, LV: left ventricle, M: mass (metastatic tumor), RA: right atrium, RCA: right coronary artery, RV: right ventricle

revealed a huge metastatic tumor over the anterior wall of the right atrium extending to the interatrial and interventricular septa (Fig. 4, 5). The right coronary artery was surrounded by a tumor, and the stents were visible within the metastatic tumor. The patient decided to spend time at home and was discharged on the 19th hospital day.

\section{Post-discharge and autopsy details}

After spending time at home for six months after PCI, the patient was re-hospitalized for poor food intake and dyspnea. Massive bilateral pleural effusion was noted, but the symptoms were unable to be relieved by pleural drainage. On the day following admission, the patient developed ven- 
tricular fibrillation and died, and his family consented to an autopsy.

The autopsy revealed residual well-differentiated squamous cell carcinoma $(\phi 4.0 \mathrm{~cm})$ in the left upper lobe invading the anterior mediastinum and the heart. The tumor invasion extended from the aortic root to the aortic arch and its main branches (brachial artery, left common cervical artery, and left subclavian artery). The pulmonary artery, superior vena cava, and right pulmonary veins were also involved in the cancer invasion.

The PCI site of the right coronary artery was patent, but the metastatic tumor had surrounded the artery and invaded the lumen. At the base of the tricuspid valve, the metastatic tumor was found to have invaded the right atrium, and metastasis to the interventricular septum was considered to have been the cause of the conduction block. (Fig. 6).

\section{Discussion}

We herein report a case of metastasis of squamous cell lung cancer to the heart, which presented with AMI. CAG revealed total occlusion of the right coronary artery at the proximal site, and emergency PCI was performed. Imaging studies, including echocardiography and CT, revealed a metastatic tumor, and 3D TTE was able to delineate the size, extent, and location of the metastatic tumor. The PCI site was surrounded by the tumor. The patient spent time at home until his death six months after PCI. An autopsy confirmed the 3D TTE findings and the invasion of the large vessels. In this patient with cardiac metastasis, 3D TTE was useful for determining the mechanism of AMI.

Cancer metastasis to the heart presents with diverse prodromes, including heart failure, arrhythmias, valve disease, and pericardial effusion (1). Patients complain of dyspnea, palpitation with bradycardia or tachycardia, or peripheral edema. These symptoms can be modified by concomitant tumor-associated anemia or hypoproteinemia (1).

Myocardial involvement due to metastasis may provoke ST-segment elevation mimicking AMI $(3,4)$, and the metastasis may occlude the coronary artery via invasion or direct compression, necessitating emergency PCI, as in the present patient (2, 5-7). Imaging studies, especially 3D TTE, were useful for obtaining information about the extent, size, and relationship of the metastatic tumor to the coronary artery.

\section{Conclusion}

We herein report a rare case of a patient who died of AMI caused by the occlusion of the coronary artery by a metastatic mass from squamous cell lung cancer. 3D TTE was useful for obtaining precise spatial information and diagnosing the cause of AMI in this patient.

The authors state that they have no Conflict of Interest (COI).

\section{References}

1. Reynen K, Kockeritz U, Strasser RH. Metastasis to the heart. Ann Oncol 15: 375-381, 2004.

2. Gong IY, Yan AT, Ko DT, et al. Temporal changes in treatments and outcomes after acute myocardial infarction among cancer survivors and patients without cancer, 1995 to 2013. Cancer 124: 1269-1278, 2018.

3. Chen J, Craft C, Panakos AW, Marhefka GD. Squamous cell carcinoma metastatic to the heart mimicking ST-elevation myocardial infarction. The Medicine Forum 17: 2016.

4. Suga T, Akuzawa N, Hatori T, Imai K, Kitahara Y, Kurabayashi M. ST segment elevation in secondary cardiac cancer: a case report and review of the literature. Int J Clin Exp Med 8: 77197727, 2015.

5. Potts JE, IIiescu CA, Mattei JCL, et al. Percutaneous coronary intervention in cancer patients: a report of the prevalence and outcomes in the United States. Eur Heart J 40: 1790-1800, 2019.

6. Roule V, Verdier L, Blanchart K, et al. Systemic review and metaanalysis of the prognostic impact of cancer among patients with acute coronary syndrome and/or percutaneous coronary intervention. BMC Cardiovas Disord 20: 38, 2020.

7. Franciosa JA, Lawrinson W. Coronary artery occlusion due to neoplasm. A rare cause of acute myocardial infarction. Arch Intern Med 128: 797-801, 1971.

The Internal Medicine is an Open Access journal distributed under the Creative Commons Attribution-NonCommercial-NoDerivatives 4.0 International License. To view the details of this license, please visit (https://creativecommons.org/licenses/ by-nc-nd/4.0/).

\section{(C) The Japanese Society of Internal Medicine Intern Med Advance Publication}

